\title{
Desarrollo de una tortilla adicionada con harinas de aguacate y nopal y su efecto en la reducción de colesterol, triglicéridos y glucosa en ratas
}

\author{
Development of an added tortilla with avocado and nopal flours and its effect on the reduction of \\ cholesterol, triglycerides and glucose in rats
}

\begin{abstract}
José Octavio Rodiles-López, Laura Patricia Arriaga-Martínez, Héctor Eduardo Martínez-Flores*, Rafael Zamora-Vega, Rosa María García-Martínez

Facultad de Químico Farmacobiología. Universidad Michoacana de San Nicolás de Hidalgo. Tzintzuntzan No. 173. Col. Matamoros. CP. 58240 Morelia, Michoacán, México.
\end{abstract}

\section{RESUMEN}

El sobrepeso, obesidad, afecciones cardiovasculares, dislipidemias, hipertensión, diabetes y cáncer son un problema mundial de salud. Se sugiere para su prevención una alimentación sana y estilo de vida saludable. En este estudio se elaboró una tortilla de maíz a partir de $90 \mathrm{~g}$ de masa con $2.5 \mathrm{~g}$ de harina de nopal y $2.5 \mathrm{~g}$ de aguacate liofilizado, y se evaluó su efecto reductor de colesterol, triglicéridos y glucosa en ratas Wistar. Se formularon tres dietas: Referencia (DR), que incluyó todos los ingredientes que los roedores requieren para su mantenimiento, Control (DC), en donde se indujo hipertrigliceridemia e hipercolesterolemia, y una dieta con la tortilla (DT) donde también se les indujo hipertrigliceridemia e hipercolesterolemia. Los animales que fueron alimentados con DT disminuyeron sus valores de colesterol total (25.8\%), triglicéridos (30.8\%), y el colesterol-LDL (72.9\%) con respecto a DC. También hubo disminución de glucosa en los animales alimentados con DT en comparación con los alimentados con DC (31.85\%) y DR (32.84\%). Los animales que fueron alimentados con DT en comparación con DC presentaron mayor concentración de lípidos en heces y mayor humedad, $56.2 \%$ y $311 \%$, respectivamente, y un $10.4 \%$ menos concentración de lípidos en hígado. La adición de harinas de aguacate y nopal fueron efectivas en reducir las concentraciones de colesterol, triglicéridos y glucosa en ratas.

Palabras Clave: Tortilla, nopal, aguacate, dislipidemias, glucosa.

\section{ABSTRACT}

Overweight, obesity, cardiovascular diseases, dyslipidemias, hypertension, diabetes and cancer are a global health problem, and for its prevention, a healthy eating and healthy lifestyle are suggested. In this study, we elaborated a corn tortilla from $90 \mathrm{~g}$ of dough with $2.5 \mathrm{~g}$ of nopal flour and $2.5 \mathrm{~g}$ of lyophilized avocado, and evaluated the reducing effect on cholesterol, triglyceride and glucose levels in male Wistar rats was evaluated. Three diets were formulated: Reference (DR), which included all the ingredients that the rodents require for their maintenance, Control (DC), where hypertriglyceridemia and hypercholesterolemia was induced, and a diet with the tortilla (DT) where they were also induced hypertriglyceridemia and hypercholesterolemia. The

\footnotetext{
*Autor para correspondencia: Héctor Eduardo Martínez-Flores

Correo electrónico: hedu65@hotmail.com
}

Recibido: 22 de agosto de 2018

Aceptado: 6 de diciembre de 2018 animals fed with DT decreased their total cholesterol (25.8\%), triglycerides (30.8\%), and LDL-cholesterol (72.9\%) values with respect to DC. There was also a decrease in glucose in the animals fed DT as compared to those fed DC (31.85\%) y DR (32.84\%). The animals fed with DT, in comparison with DC, showed a greater concentration of feces lipids and higher moisture, $56.2 \%$ and $311 \%$, respectively, and $10.4 \%$ less concentration of lipid in the liver. The addition of avocado and nopal flours was effective in reducing the concentrations of cholesterol, triglycerides and glucose in rats.

Keywords: Tortilla, nopal, avocado, dyslipidemias, glucose.

\section{INTRODUCCIÓN}

El sobrepeso y la obesidad son la acumulación anormal de grasa que puede ser perjudicial para la salud. En 2016, en el mundo más de 1,900 millones de adultos de 18 o más años tenían sobrepeso, y de los cuales, más de 600 millones eran obesos. En México, las cifras son devastadoras, cerca del $72.5 \%$ de la población adulta presenta sobrepeso u obesidad. El sobrepeso y obesidad son el principal factor de riesgo de enfermedades no trasmisibles, como las cardiovasculares (cardiopatías y accidentes cerebrovasculares), diabetes, trastornos del aparato locomotor como la osteoartritis, y algunos tipos de cáncer (endometrio, mama, ovarios, próstata, hígado, vesícula biliar, riñones y colon) (ENSANUT, 2016; OMS, 2016). Las enfermedades cardiovasculares generalmente se asocian con una mala alimentación, debido al consumo de grasas de origen animal que contribuye con lípidos que contienen ácidos grasos saturados y colesterol, aunado a un estilo de vida sedentario. Por otro lado, la diabetes se caracteriza por niveles altos de glucosa en sangre, tanto en la población adulta, como en adolescentes y niños (Sánchez et al., 2015).

Con base en ello, la población mundial debe poner mayor atención en tener una alimentación sana como un componente fundamental en el tratamiento y/o prevención de las enfermedades cardiovasculares y diabetes. Ciertas propiedades funcionales de bienestar a la salud pueden obtenerse a partir de alimentos vegetales que contienen compuestos bioactivos. Existen alimentos que cumplen con las características de poseer compuestos bioactivos como son el nopal y el aguacate, o combinaciones de éstos (De Smet, 2002). 
La especie de nopal más conocida y comercializada es la Opuntia ficus-indica, la cual produce frutas comestibles dulces, llamadas tunas, y sus cladodios, también denominadas pencas, son consumidos como vegetales frescos, siendo una materia vegetal extensamente consumida en México $y$ en el sur de los Estados Unidos (Feugang et al., 2006). El nopal ha sido utilizado con fines medicinales desde la época precolombina, extendiéndose a otros países como parte de la medicina alternativa por sus efectos hipoglucemiantes en el tratamiento de la diabetes mellitus tipo 2 y por sustancias bioactivas como antioxidantes (flavonoides, flavonoles, y carotenos), vitaminas del complejo B, A y C, y minerales como el $\mathrm{Ca}$, Mg y Fe, así como fibra dietética. La fibra del nopal representa más del $50 \%$ de sus sólidos totales, siendo de particular interés el mucílago, que al ponerse en contacto con agua forma una solución de elevada viscosidad. Esta capacidad gelificante es la responsable de los múltiples efectos fisiológicos de la fibra, como son tanto la disminución de la glucemia postprandial, como de colesterol y triglicéridos, por lo que se ha propuesto utilizarla para tratar dichas enfermedades (Medina et al., 2011).

Otra planta con propiedades benéficas es el aguacate, originario de México y Centroamérica, perteneciente a la familia Lauraceae, género Persea. El aguacate es considerado como una de las fuentes nutricionales más importantes debido principalmente a sus componentes nutracéuticos que pueden tener un efecto terapéutico en la obesidad e hipercolesterolemia (Pahua et al., 2014). Este fruto se caracteriza por su alta cantidad de fibra, $6.7 \%$, en donde el $75 \%$ es fibra insoluble y el $25 \%$ fibra soluble; además de lípidos, $14.7 \%$, de los cuales el $71 \%$ son ácidos grasos monoinsaturados en una matriz a base de agua, $13 \%$ de ácidos grasos poliinsaturados y 16\% de ácidos grasos saturados (Dreher y Davenport, 2013; USDA, 2018). El consumo de aguacate se ha relacionado con la disminución de algunos factores de riesgo cardiovasculares, así como en la mejora de la calidad dietética mediante el aumento del consumo de ácidos grasos monoinsaturados, fibra dietética, los minerales $\mathrm{Mg}$ y $\mathrm{K}$, y las vitaminas $\mathrm{E}$ y $\mathrm{K}$. Investigaciones recientes han demostrado que el aguacate puede tener un efecto hipocolesterolémico y ser útil en el tratamiento de la hipertensión arterial, así como la diabetes mellitus tipo 2 (Kuo, 2013; Wang et al., 2015). Con base en lo anterior se ha planteado el objetivo de evaluar las propiedades de una tortilla de maíz adicionada con harinas de aguacate y nopal para el control de algunos parámetros lipídicos y de glucosa utilizando ratas Wistar.

\section{MATERIALES Y MÉTODOS Materias primas}

Se usó aguacate liofilizado variedad Hass proporcionado por la empresa SíoSí Alimentos SAPI de CV, localizada en Morelia, Michoacán, México, y harina de nopal de la empresa Comerspro del Pacífico, Morelia, Michoacán, México. Las tortillas fueron elaboradas manualmente usando masa de maíz nixtamalizada, adicionada con las harinas de nopal y aguacate, a la cual se le denominó tortilla funcional. La masa nixtamalizada se obtuvo de la siguiente manera: $1 \mathrm{~kg}$ de maíz fue colocado en $3 \mathrm{~L}$ de agua con $1 \%$ de hidróxido de calcio $(p / p)$, y se colocó en ebullición por 40 min. Posteriormente, los granos de maíz cocidos se dejaron en reposo por $16 \mathrm{~h}$. Enseguida, se lavaron los granos de maíz con agua para eliminar el exceso de cal y sólidos desprendidos. Los granos de maíz nixtamalizados fueron molidos en un molino de piedras hasta obtener la masa nixtamalizada. La mezcla de vitaminas y minerales para las dietas de los animales se obtuvieron de Harlan México, y que corresponden a una mezcla de vitaminas, AIN-93-VX, y minerales AIN-93G-MX; el colesterol fue adquirido de Sigma-Aldrich, México; y el aceite de coco utilizado fue de la marca LouAna Oils ${ }^{\oplus}$, Ventura Foods, California, USA.

\section{Análisis químico proximal}

Los estudios de composición química de la tortilla de maíz, y de las harinas de aguacate y nopal se realizaron de acuerdo con los métodos de la AOAC (1990) e incluyeron humedad, lípidos, proteína, y cenizas; la fibra dietética se evaluó por el método de Prosky et al. (1998). El valor de Extracto Libre de Nitrógeno (E.L.N.) se obtuvo de la siguiente fórmula $=100$ - contenidos de proteína + cenizas + lípidos + fibra dietética.

\section{Formulación tortilla-nopal-aguacate}

Se realizaron dos formulaciones para la elaboración de la tortilla, usando como base maíz nixtamalizado, aguacate y nopal. La formulación 1 (F1) se preparó con $100 \mathrm{~g}$ de masa de maíz, $2.5 \mathrm{~g}$ de aguacate liofilizado y $2.5 \mathrm{~g}$ de nopal deshidratado. La formulación 2 (F2) se elaboró con $100 \mathrm{~g}$ de masa de maíz, $5.0 \mathrm{~g}$ de aguacate liofilizado y $5.0 \mathrm{~g}$ de nopal deshidratado. Las dos formulaciones fueron evaluadas sensorialmente mediante comparación directa de las mismas usando 20 panelistas no entrenados, en donde se compararon ambas tortillas en una prueba ciega, en la que se les pidió a los panelistas que dijeran cuál de las dos tortillas era más de su agrado, con el fin de seleccionar la formulación con mejor aceptación.

\section{Formulación de dietas para el ensayo biológico}

Se formularon tres dietas: 1) DR, dieta de referencia; 2) DC, dieta control; 3) DT, dieta a base de la tortilla adicionada con las harinas de aguacate y nopal. La DR consistió en una dieta estándar de mantenimiento para roedores sanos, formulada de acuerdo con lo recomendado por Reeves et al. (1993), en donde la concentración de lípidos fue del $4 \%$. La DC correspondió a la dieta donde se promovió un cuadro de hipercolesterolemia e hipertrigliceridemia, inducida por la adición de $1.5 \%$ de colesterol y $10 \%$ de aceite de coco, respectivamente. Esta fórmula fue igual a la DR más la adición del colesterol y del aceite de coco. El aceite de coco se utiliza como una fuente para incorporar a la dieta un mayor contenido de ácidos grasos saturados y aumentar el cuadro de dislipidemia, de acuerdo a lo descrito en Martínez Flores 
et al. (2004). Por otro lado, la DT consistió en la tortilla de maíz adicionada con las harinas de aguacate y nopal, así como $1.5 \%$ de colesterol y $10 \%$ de aceite de coco, y se formuló de manera tal que su contenido de lípidos fuera igual a DR y DC; de tal forma que los lípidos fueran aportados por la tortilla, y en donde los demás nutrientes se ajustaron para que fueran lo más cercano posible a DR y DC. En la Tabla 1 se muestran las formulaciones de dichas dietas.

Tabla 1. Formulación de dietas experimentales.

Table 1. Formulation of experimental diets.

\begin{tabular}{lccc}
\hline $\begin{array}{l}\text { Ingrediente } \\
(\mathbf{g})\end{array}$ & $\begin{array}{c}\mathbf{D R}^{\mathbf{1}} \\
\text { (g/100 dieta) }\end{array}$ & $\begin{array}{c}\text { DC } \\
\text { (g/100 dieta) }\end{array}$ & $\begin{array}{c}\text { DT } \\
\text { (g/100 dieta) }\end{array}$ \\
\hline Almidón & 66.5 & 65.0 & 6.1 \\
\hline Celulosa & 5.0 & 5.0 & - \\
\hline Caseína & 10.0 & 10.0 & 7.7 \\
\hline Aceite de Soya & 4.0 & 4.0 & - \\
\hline Minerales & 3.5 & 3.5 & 2.4 \\
\hline Vitaminas & 1.0 & 1.0 & 1.0 \\
\hline Tortilla & - & - & 71.3 \\
\hline Sacarosa & 10.0 & 0 & 0 \\
\hline Colesterol & - & 1.5 & 1.5 \\
\hline Aceite de Coco & - & 10.0 & 10.0 \\
\hline
\end{tabular}

'DR: Dieta de referencia. DC: Dieta control. DT: Dieta a base de tortilla.

\section{Ensayo biológico}

Se usaron 12 ratas macho Wistar adultos jóvenes proporcionadas por el Bioterio de la Facultad de Ciencias de la Universidad Nacional Autónoma de México, Campus Juriquilla, Querétaro, México. Las ratas se mantuvieron en un periodo de adaptación por 7 días, y se les dio alimento comercial Chow 5001. El peso promedio de animales por grupo de prueba al iniciar el periodo de experimentación fue de $449.4 \pm 25.3 \mathrm{~g}$. Las ratas fueron colocadas en jaulas individuales en un ambiente aislado y controlado de ruido para evitar un estado de estrés. El estudio se realizó en el Bioterio de la Facultad de Químico Farmacobiología de la Universidad Michoacana de San Nicolás de Hidalgo. La temperatura al interior del bioterio fue de $22^{\circ} \mathrm{C}$, con ciclos de luz y oscuridad de $12 \mathrm{~h}$.

Los animales fueron divididos en tres lotes distintos de cuatro animales por lote, y pesados individualmente antes de empezar el experimento. Los roedores se distribuyeron de manera tal que al final cada lote experimental tuviera el mismo peso como bloque de prueba. El experimento duró 21 días, tiempo en el que a los animales se les suministró agua ad libitum. Al inicio y al final del experimento se tomó el peso de cada rata y se calculó el porcentaje de aumento de peso de las ratas al término de los 21 días, empleando la siguiente fórmula:

Ecuación 1. Aumento de peso en animales \%.

$\%$ Aumento de Peso $=($ Peso Final-Peso Inicial $) \times 100$ Consumo
Asimismo, se midió el consumo diario de alimento por parte de los roedores. Por día se colocaban $20 \mathrm{~g}$ de alimento dentro de un recipiente. Al día siguiente se pesó el alimento que sobró, y se recolectó el alimento que tiraron. La suma del alimento sobrante más el que tiraron fueron sumados y este valor se restó a los $20 \mathrm{~g}$. Adicionalmente, las heces fueron colectadas durante siete días consecutivos una semana antes del sacrifico para la medición de humedad y lípidos totales. Las heces fueron secadas en estufa a $40^{\circ} \mathrm{C}$ y pulverizadas en mortero. Una noche antes de ser sacrificados, se les retiró el alimento y el agua. El sacrificio se realizó por dislocación cervical, de acuerdo con lo establecido por la Norma Oficial Mexicana NOM-062-ZOO-1999. Una vez sacrificados los roedores se procedió a extraer muestra sanguínea vía punción cardíaca para hacer el correspondiente análisis sanguíneo. Asimismo, al final del experimento se tomaron tres animales al azar de cada bloque de prueba para la extracción del hígado. Las muestras se pesaron, lavaron, trituraron, secaron en estufa convencional a $50^{\circ} \mathrm{C}$ y guardadas en recipientes cerrados hasta su posterior análisis.

\section{Análisis en tejidos sanguíneo, en hígado y heces}

La sangre colectada de cada animal se centrifugó a 4,000 rpm por $15 \mathrm{~min}$, posteriormente se almacenó a $4{ }^{\circ} \mathrm{C}$ hasta su uso. Se realizaron los análisis de glucosa y de perfil lipídico que incluyeron el colesterol total (CT), triglicéridos (TG), colesterol HDL (C-HDL), y colesterol LDL (C-LDL), con kits enzimáticos (Wiener laboratorios S.A.I.C., Rosario, Argentina), medidos en espectrofotómetro UV/Vis (Marca VELAB modelo VE-5600UV. Científica Vela Quin S.A. de C.V. Ciudad de México, México). A las heces colectadas de los animales se les midió humedad por el método de la AOAC secando las muestras por un periodo de 24 horas a $40^{\circ} \mathrm{C}$. Los lípidos en heces fueron extraídos usando un equipo de Soxleth, extrayendo con éter de etílico durante un período de 6 horas. La cuantificación de lípidos por esta forma se hizo de forma gravimétrica, utilizándose el método de la AOAC. El contenido de lípidos en hígado se realizó con la misma metodología utilizada para la cuantificación de lípidos en heces.

\section{Análisis estadístico}

Todos los datos fueron procesados usando la prueba de Tukey Kramer con $p<0.05$, con el software estadístico JMP6 (SAS Institute, Cary, NC, USA, 2004).

\section{RESULTADOS Y DISCUSIÓN}

Se desarrollaron dos formulaciones de tortillas adicionadas con harina de nopal y aguacate. F1, que contenía $2.5 \%$ de harina de aguacate y $2.5 \%$ de harina de nopal; y F2, que contenía $5 \%$ de harina de aguacate y $5 \%$ de harina de nopal. La primera etapa consistió en hacer un análisis sensorial, con la finalidad de seleccionar la mejor tortilla aceptada por el panel, y con base en ello continuar con el estudio del ensayo biológico. 


\section{Evaluación sensorial}

Para el estudio del análisis sensorial, se evaluaron los parámetros de color, olor, sabor y textura, usando 20 panelistas no entrenados. Tal como se observa en la Figura 1, los evaluadores tuvieron una amplia aceptación por la tortilla formulada con $2.5 \%$ de harina de aguacate y $2.5 \%$ de harina de nopal (tortilla F1) comparado con la tortilla que tenía 5\% (tortilla F2) de cada una de las harinas referidas. En los parámetros de color, olor y sabor la aceptación fue de un 70\% con la tortilla que contenía $2.5 \%$ de cada una de las harinas adicionadas y $60 \%$ en el parámetro de textura, comparado con la tortilla preparada con $5 \%$ de cada una de las harinas adicionadas. Los evaluadores mencionaron que la tortilla F2 fue muy quebradiza, de color verde muy oscuro poco atractivo, de sabor amargo y olor a tierra. Con base en este criterio de evaluación de parámetros sensoriales de las tortillas, se decidió seleccionar la tortilla F1 para continuar con el resto de la experimentación.

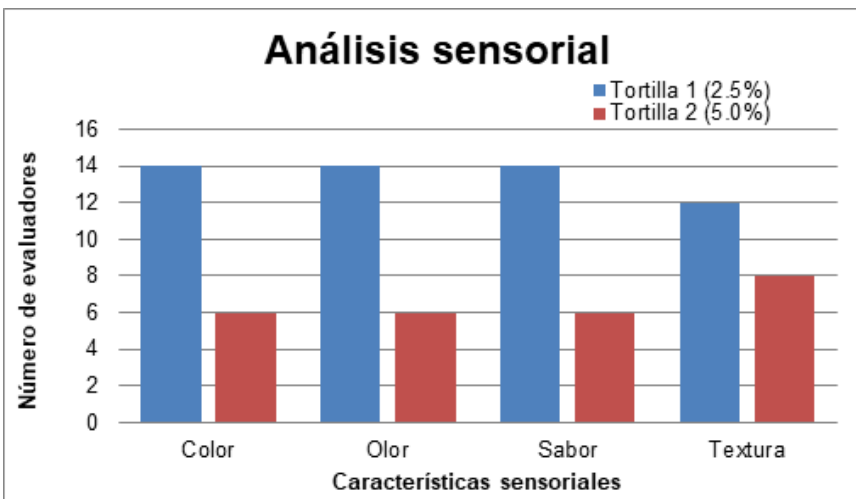

Figura 1. Evaluación sensorial de las tortillas de maíz. Tortilla 1: adicionada con $2.5 \mathrm{~g}$ de harinas de aguacate y nopal. Tortilla 2: adicionada con $5 \mathrm{~g}$ de harinas de aguacate y nopal.

Figure 1. Sensory evaluation of corn tortillas. Tortilla 1: added with $2.5 \mathrm{~g}$ of avocado and cactus flours. Tortilla 2: added with $5 \mathrm{~g}$ of avocado and nopal flours.

\section{Análisis químico proximal}

Se determinó la composición química de las harinas de nopal y de aguacate, además de la tortilla adicionada con las harinas de nopal $(2.5 \%)$ y aguacate $(2.5 \%)$ como se muestra en la Tabla 2 . Se observa que el aguacate liofilizado aporta una gran cantidad de lípidos $(60.46 \%)$, de fibra dietética (19.42\%) y de cenizas (7.11\%). La harina de nopal, a su vez contiene grandes cantidades de fibra dietética (37.45\%), proteína (22.45\%) y cenizas (19.42\%). Cuando se adicionaron las harinas de aguacate y de nopal los contenidos de cenizas, lípidos, proteína y fibra dietética aumentaron en un 60.74 , $25.51 \%, 14.08 \%$ y $11.72 \%$, respectivamente, y una disminución del extracto libre de nitrógeno del $6.75 \%$ en relación a la tortilla control.

A partir de la composición química de la tortilla se formularon las dietas de los animales en estudio.

\section{Ensayo biológico}

Se usaron ratas Wistar para evaluar los beneficios de la tortilla adicionada con las harinas de aguacate y nopal.
Es importante señalar que los animales que consumieron la tortilla también consumieron el colesterol y el aceite de coco, es decir, eran animales a los que se les indujo y mantuvo el cuadro de hipercolesteremia e hipertrigliceridemia, con la finalidad de observar si los animales con cuadro de dislipidemia podrían mejorar su salud por el consumo de la tortilla, aun con una dieta alta en colesterol y triglicéridos.

Tabla 2. Análisis químico proximal de las harinas de aguacate, de nopal y de las tortillas.

Table 2. Proximate chemical analysis of avocado, nopal and tortillas flours.

\begin{tabular}{lcccc}
\hline $\begin{array}{l}\text { Componente } \\
\text { Químico } \\
\text { (g/100 g) }\end{array}$ & $\begin{array}{c}\text { Aguacate } \\
\text { liofilizado } \\
(\%)\end{array}$ & $\begin{array}{c}\text { Nopal } \\
\text { deshidratado } \\
(\%)\end{array}$ & $\begin{array}{c}\text { Tortilla }^{1} \\
\text { Funcional } \\
(\%)\end{array}$ & $\begin{array}{c}\text { Tortilla } \\
\text { Control } \\
\text { (\%) }\end{array}$ \\
\hline Fibra Dietética $^{2}$ & 19.42 & 37.45 & 12.77 & 11.43 \\
\hline Proteína $^{2}$ & 3.90 & 22.42 & 5.59 & 4.90 \\
\hline Lípidos $^{2}$ & 60.46 & 1.50 & 9.79 & 7.80 \\
\hline Cenizas $^{2}$ & 7.11 & 19.42 & 2.62 & 1.63 \\
\hline E.L.N. $^{2}$ & 9.11 & 19.21 & 69.23 & 74.24 \\
\hline
\end{tabular}

${ }^{1}$ La tortilla contiene $2.5 \%$ de polvo de aguacate y $2.5 \%$ de polvo de nopal. E.L.N. Extracto libre de nitrógeno.

${ }^{2}$ Los valores están expresados en base seca.

Se determinó el peso inicial, peso final y el porcentaje de aumento de peso para cada una de las ratas alimentadas con las dietas, así como consumo de alimento, tal como se muestra en la Tabla 3. De acuerdo con los resultados obtenidos se observó que no hubo diferencia significativa $(\mathrm{p}<$ 0.05 ) en el peso inicial de los animales alimentados con las tres dietas. El rango de peso inicial fue de $445 \mathrm{~g}$ para las ratas alimentadas con DC y de $457 \mathrm{~g}$ para las alimentadas con DR. Tampoco se observó diferencia significativa $(p<0.05)$ en el peso final de los mismos, el cual estuvo en un rango de 518 g para los roedores alimentados con la DR y de $541 \mathrm{~g}$ para las alimentadas con la DT. En cuanto al aumento de peso porcentual tampoco existió diferencia significativa $(p<0.05)$ teniendo un rango de $11.7 \%$ de la DR y de $15.5 \%$ para las ratas alimentadas con DC, lo que implicó un aumento de peso del $32.48 \%$. Por otro lado, los animales alimentados con DT tuvieron un aumento de peso del $13.1 \%$ con respecto a los roedores alimentados con DR, y una disminución con respecto a DC. Los animales alimentados con DT disminuyeron $15.4 \%$ en peso con respecto a DC, es decir, disminuyeron de

Tabla 3. Peso y ganancia de peso de animales y consumo de alimento de ratas alimentadas con las dietas experimentales.

Table 3. Weight and weight gain of animals and feed intake of rats fed with the experimental diets.

\begin{tabular}{lccc}
\hline & DR $^{2}$ & DC & DT \\
\hline Peso Inicial (g) & $457^{\text {a1 }}$ & $445^{\text {a }}$ & $447^{\text {a }}$ \\
\hline Peso Final (g) & $518^{\text {a }}$ & $526^{\text {a }}$ & $541^{\text {a }}$ \\
\hline Consumo de Alimento (g) & $525^{\mathrm{b}}$ & $528^{\mathrm{b}}$ & $719^{\mathrm{a}}$ \\
\hline Aumento de Peso (\%) & $11.7^{\mathrm{a}}$ & $15.5^{\mathrm{a}}$ & $13.1^{\mathrm{a}}$ \\
\hline
\end{tabular}

'Letras iguales en la misma fila no presentan diferencia significativa (p>0.05). DR: Dieta de referencia.

${ }^{2}$ DC: Dieta control. DT: Dieta a base de tortilla. 
peso por el consumo de la tortilla funcional. Pee et al. (2014), mencionan la eficacia de la fibra dietética en la reducción del peso corporal, y que puede atribuirse a una menor absorción de las grasas y por lo tanto menor aporte de calorías al organismo.

Las dietas DC y DR contenían la misma cantidad de fibra que DT, sin embargo, ésta última fue exclusivamente celulosa, es decir, una fibra insoluble, mientras que DT fue una fibra dietética, mezcla de fibra soluble e insoluble. La fibra soluble absorbe grasas y azúcares, capta mucha agua y forman soluciones viscosas de gran volumen en el intestino delgado, y constituyen un sustrato fermentable para los microorganismos intestinales. El consumo de este tipo de fibra está asociado con la disminución de glucosa, triglicéridos y colesterol en sangre. La fibra insoluble favorece el aumento del tamaño de la materia fecal, incrementando la velocidad del tránsito y la distención intestinal tal como lo menciona Gil (2010), Dreher y Davenport (2013) y Seema (2014).

\section{Perfil de lípidos y glucosa}

A las muestras sanguíneas de los animales se les determinó un perfil lipídico, en donde se evaluaron los contenidos de colesterol total (CT), colesterol-HDL, colesterol-LDL, triglicéridos (TG), y glucosa. Los resultados se muestran en la Tabla 4. Se observó un incremento del $63.9 \%$ del CT de los roedores alimentados con DC comparados con los alimentados con DR, debido a que la dieta DC estuvo adicionada con colesterol. Por otro lado, la dieta con tortilla disminuyó $25.8 \%$ la concentración de CT en muestras de sangre de los roedores con respecto a los que consumieron DC.

Asimismo, se observó un incremento en la concentración de triglicéridos en los roedores alimentados con DC en comparación con $\mathrm{DR}$, debido a la adición de aceite de coco como vehículo para presentar un cuadro de hipertrigliceridemia. El incremento fue del $80.0 \%$. Nuevamente se observó una disminución en este parámetro en la dieta a base de la DT con respecto a DC, siendo de $30.8 \%$. Por lo tanto, la tortilla de maíz adicionadas con aguacate liofilizado y harina de nopal disminuyó las concentraciones de triglicéridos sanguíneos.

Tabla 4. Análisis sanguíneos en ratas alimentadas con las dietas de referencia (DR), control (DC) y de tortilla (DT).

Table 4. Blood tests in rats fed the reference (DR), control (DC) and tortilla (DT) diets.

\begin{tabular}{lccc}
\hline $\begin{array}{l}\text { Parámetro } \\
\text { sanguíneo }\end{array}$ & DR & DC & DT \\
\hline $\begin{array}{l}\text { Colesterol Total } \\
\text { (mg/dL) }\end{array}$ & $51.97 \pm 2.55^{\mathrm{b} 1,2}$ & $85.17 \pm 9.69^{\mathrm{a}}$ & $63.17 \pm 4.36^{\mathrm{b}}$ \\
$\begin{array}{l}\text { Colesterol HDL } \\
\text { (mg/dL) }\end{array}$ & $37.53 \pm 6.11^{\mathrm{a}}$ & $36.37 \pm 1.91^{\mathrm{a}}$ & $33.93 \pm 2.76^{\mathrm{a}}$ \\
$\begin{array}{l}\text { Colesterol LDL } \\
\text { (mg/dL) }\end{array}$ & $6.00 \pm 1.00^{\mathrm{b}}$ & $44.33 \pm 16.44^{\mathrm{a}}$ & $12.00 \pm 3.46^{\mathrm{b}}$ \\
\hline $\begin{array}{l}\text { Triglicéridos (mg/dL) } \\
\text { Glucosa (mg/dL) }\end{array}$ & $121.40 \pm 20.0^{\mathrm{b}}$ & $218.50 \pm 37.4^{\mathrm{a}}$ & $151.27 \pm 29.6^{\mathrm{a}, \mathrm{b}}$ \\
\hline
\end{tabular}

1 Letras iguales en la misma fila no presentan diferencia significativa ( $p>0.05)$.

2 Los resultados están expresados como el promedio \pm la desviación estándar.
Por otro lado, en relación con la fracción de colesterolLDL, se notó un gran aumento en la muestra sanguínea de los animales alimentados con DC con respecto a los que consumieron DR, alrededor del $638.83 \%$, y una disminución en las muestras sanguíneas de los roedores alimentados con DT con respecto a la DC, siendo de un $72.9 \%$. Esto implica que la tortilla adicionada con harina de nopal y aguacate liofilizado bajó la concentración de la fracción de colesterol-LDL. Butterweck et al. (2011), trabajaron con ratas alimentadas con una dieta a base de cladodios de nopal y demostraron una reducción del $34 \%$ en la concentración de la fracción de colesterol-LDL, concluyendo que existió un efecto potencialmente beneficioso de la fibra del nopal sobre el cuadro de hipercolesterolemia. Por su parte, Padmanabhan y Arumugam (2014), realizaron un estudio con ratas macho Sprague Dawley, alimentándolas vía oral en dosis de $100 \mathrm{mg} / \mathrm{kg}$ de peso corporal con un extracto de aguacate (Persea americana) liofilizado, cuyos resultados indicaron que el índice de masa corporal, grasa total y el índice de adiposidad disminuyeron significativamente en las ratas administradas con el aguacate, a diferencia de las ratas alimentadas con el control. Asimismo, las concentraciones de la fracción de colesterolLDL y peróxidos lipídicos fueron significativamente mayores en el grupo control que con el aguacate.

En este estudio no se indujo un cuadro de hiperglucemia en los animales control, DC. Sin embargo, en relación con la glucosa sanguínea evaluada se pudo observar que entre los animales alimentados con la dieta con tortilla con respecto a DR y DC, no hubo diferencia significativa $(p>0.05)$ entre estas dos últimas, tal como se muestra en la Tabla 4. Cabe mencionar que en los roedores alimentados con la dieta a base de tortilla (DT) se logró una disminución significativa ( $p$ $<0.05$ ) del $24.72 \%$ comparándolas con las ratas alimentadas con la dieta de referencia (DR). Hahm et al. (2011), realizaron un estudio con ratas Wistar diabéticas, reportando que cuando fueron tratadas con cladodio liofilizado del género Opuntia disminuyeron significativamente la concentración de glucosa en ayunas, atribuyéndosele principalmente a un aumento en la producción de insulina. Por otro lado, Morán et al. (2012), mencionaron que los cladodios deshidratados de O. ficus-indica mejoraron la sensibilidad a la insulina hepática, reduciendo consecuentemente la gluconeogénesis hepática en ratas Zucker obesas, ya que la gluconeogénesis es modulada por la vía de señalización de la insulina. Asimismo, Núñez et al. (2013), reportaron que las harinas de cladodio (O. ficus-indica) obtenidas en diferentes etapas de maduración, mostraron diferentes efectos antihiperglucémicos e hipoglucémicos, debido a mecanismos de atrapamiento de glucosa y retraso de la absorción intestinal.

\section{Análisis en hígado y heces}

Asimismo, se realizó un estudio de lípidos en hígado y heces, así como de humedad en heces, los cuales se muestran en la Tabla 5. Hubo aumento significativo $(p<0.05)$ del $222.17 \%$ en la concentración de lípidos en hígado de aqueIlos animales alimentados con la DC con respecto a DR, es 
decir, a las que se les indujo el cuadro de hipercolesteremia e hipertrigliceridemia. Por otro lado, hubo una disminución significativa $(p<0.05)$ de $10.4 \%$ de los lípidos en el hígado de los animales que consumieron la tortilla con respecto a DC. El efecto de disminución de lípidos en hígado de ratas alimentadas con la dieta a base de tortilla funcional fue debido posiblemente a que presenta un $11.72 \%$ más de fibra dietética en relación a la tortilla control. Lo anterior permite que la fibra dietética de la tortilla funcional sea más efectiva para atrapar lípidos en el tracto gastrointestinal, evitando la absorción al interior del organismo, y excretándola junto con las heces, tal como también fue reportado por Martínez-Flores et al. (2004) cuando realizó experimentos estudiando el efecto de la fibra de avena y de almidón resistente en la disminución de colesterol y triglicéridos evaluado en hámsteres. Tabeshpour et al. (2017), realizaron un estudio en ratas, donde reportaron en páncreas, que una dieta a base de aguacate mejoró los biomarcadores de lípidos séricos, como son CT, TG, C-LDL y C-HDL, regulando la hidrólisis de diferentes lipoproteínas y su absorción selectiva y metabolismo por diferentes tejidos, además de una disminución de la lipogénesis hepática y estrés oxidativo.

Tabla 5. Contenido de lípidos en hígado y de lípidos y humedad en heces de ratas alimentadas con las dietas de refeencia (DR), control (DC) y tortilla funcional (DT).

Table 5. Lipid content in liver and lipid and moisture in feces of rats fed the refeeding (DR), control (DC) and functional tortilla (DT) diets.

\begin{tabular}{lccc}
\hline & DR & DC & DT \\
\hline Lípidos en hígado (\%) $^{14.07 \pm 0.55^{\mathrm{c} 1,2}}$ & $45.33 \pm 1.02^{\mathrm{a}}$ & $40.63 \pm 0.06^{\mathrm{b}}$ \\
Lípidos en heces (\%) & $1.70 \pm 0.10^{\mathrm{c}}$ & $17.80 \pm 0.50^{\mathrm{b}}$ & $27.80 \pm 0.36^{\mathrm{a}}$ \\
\hline Humedad en heces (\%) & $13.50 \pm 0.20^{\mathrm{b}}$ & $10.70 \pm 1.15^{\mathrm{b}}$ & $44.03 \pm 3.51^{\mathrm{a}}$ \\
\hline
\end{tabular}

${ }^{1}$ Letras iguales en la misma fila no presentan diferencia significativa ( $p>0.05)$.

${ }^{2}$ Los resultados están expresados como el promedio \pm la desviación estándar.

Por otro lado, en nuestro estudio existió un aumento significativo $(p<0.05)$ en la concentración de lípidos en heces en los animales a los que se les indujo la hipercolesteremia e hipertrigliceridemia con respecto al control, debido al aumento de lípidos en la dieta. Por otro lado, se observó un aumento en los lípidos en heces de los animales que consumieron la tortilla funcional (DT) adicionada con las harinas de aguacate y nopal, con respecto a la DC, y cuyo aumento fue de $56.2 \%$.

Además, se observó un mayor contenido de humedad en las heces de los animales que consumieron la tortilla funcional. El aumento fue mayor al $311 \%$ con respecto a la de los animales a los que se les indujo el cuadro de hipercolesteremia e hipertrigliceremia (DR). La humedad en heces de los animales alimentados con tortilla fue mayor aún en los animales con la DC, lo que implica que la tortilla funcional aumentó en general la humedad en heces, debido también al mayor contenido de fibra dietética de la tortilla funcional en relación a la tortilla control.

\section{CONCLUSIONES}

La tortilla formulada con harina nixtamalizada de maíz adicionada con $2.5 \%$ de harina de nopal y $2.5 \%$ de harina de aguacate liofilizado permitió observar una disminución significativa de colesterol total, fracción de colesterol-LDL y triglicéridos en los animales alimentados con la tortilla con respecto a los de control positivo, es decir, a los que se les indujo un cuadro de hipercolesterolemia e hipertrigliceridemia. También se observó una disminución de glucosa en los animales con respecto a los animales control. Asimismo, existió un aumento en lípidos y humedad en heces en los animales que fueron alimentados a base de tortilla, y una disminución en lípidos en hígado con respecto a los animales de control positivo. La tortilla aquí formulada puede ser considerada como una tortilla de tipo funcional, es decir, un alimento funcional, y pudiera ser una alternativa viable para prevención de enfermedades que se generan a partir del elevado contenido de colesterol, triglicéridos y azúcar.

\section{AGRADECIMIENTOS}

Se agradece a la empresa SíoSí Alimentos SAPI de CV., Morelia, México, por proporcionar las muestras de aguacate liofilizado.

\section{REFERENCIAS}

AOAC. 1990. Official Methods of Analysis. $15^{\text {th }}$ Edition. Association of Official Analytical Chemists. Washington DC. USA.

Butterweck, V., Semlin, L., Feistel, B., Pischel, I., Bauer, K. y Verspohl, E. 2011. Comparative evaluation of two different Opuntia ficus indica extracts for blood sugar lowering effects in rats. Phytotherapy Research. 25: 370-375.

De Smet, P. 2002. Herbal remedies. The New England Journal of Medicine. 347: 2046-2056.

Dreher, M. y Davenport, A. 2013. Hass avocado composition and potential health effects. Critical Reviews Food Science and Nutrition 53: 738-750.

ENSANUT. 2016.

http://promocion.salud.gob.mx/dgps/descargas $1 /$ doctos_2016/ensanut_mc_2016-310oct.pdf

Feugang, J., Konarski, P., Zou, D., Stintzing, F. y Zou, C. 2006. Nutritional and medicinal use of cactus pear (Opuntia spp.) cladodes and fruits. Frontiers in Bioscience. 11: 2574-2589. PMID: 16720335.

Gil, A. 2010. Tratado de Nutrición. Tomo 2. Composición y calidad nutritiva de los alimentos. Capítulo 18. Complementos alimenticios. Editorial Panamericana. $2^{\circ}$ Edición. 483-484.

Hahm, S., Park, J. y Son, Y. 2011. Opuntia humifusa stems lower blood glucose and cholesterol levels in streptozotocininduced diabetic rats. Nutrition Research. 31: 479-487.

Kuo, S. 2013. The interplay between fiber and the intestinal microbiome in the inflammatory response. Advances in Nutrition. 4: 16-28.

Martinez-Flores, H.E., Kil Chang Y., Martinez-Bustos, F. y Sgarbieri, V. 2004. Effect of high fiber products on blood lipids and lipoproteins in hamsters. Nutrition Research. 24: 85-93.

Medina, T., Vernon, C., Gallegos, I., Rocha, G., Herrera, V., Calderas, F. y Jiménez, A. 2011. Study of the antioxidant properties of 
extracts obtained from nopal cactus (Opuntia ficus-indica) cladodes after convective drying. Journal Science Food and Agriculture. 91: 1001-1005.

Morán, R., Avila, N., Tovar, A., Pedraza, C., López, R. y Torres, N. 2012. Opuntia ficus indica (nopal) attenuates hepatic steatosis and oxidative stress in obese Zucker ( $\mathrm{fa} / \mathrm{fa}$ ) rats. Journal Nutrition. 142: 1956-1963.

NOM-062-ZOO-1999. Norma Oficial Mexicana. 1999. Especificaciones técnicas para la producción, cuidado y uso de los animales de laboratorio. Diario Oficial de la Federación. México.

Nuñez, L., Paredes, L. y Reynoso, C. 2013. Functional and hypoglycemic properties of nopal cladodes (O. ficus indica) at different maturity stages using in vitro and in vivo tests. Journal Agricultural and Food Chemistry. 61: 10981-10986.

OMS. Organización Mundial de la Salud. 2016.

http://www.who.int/mediacentre/factsheets/fs311/es/

Padmanabhan, M. y Arumugam, G. 2014. Effect of Persea americana (avocado) fruit extract on the level of expression of adiponectin and PPAR- $\gamma$ in rats subjected to experimental hyperlipidemia and obesity. Journal of Complementary and Integrative Medicine. 11: 107-119.

Pahua, R., Garduño, S., Dorantes, Á., Chamorro, C., Herrera, M., Osorio, E. y Ortiz, M. 2014. Reduced calorie avocado paste attenuates metabolic factors associated with a hypercholesterolemic high fructose diet in rats. Plant Foods for Human Nutrition. 69(1): 18-24.

Pee, W., Kai, Z., Gruenwald, J. y Uebelhack, R. 2014. A review of the efficacy and safety of litramine IQP-G-002AS, an Opuntia ficus-indica derived fiber for weight management. Evidence Based Complementary and Alternative Medicine. Article ID 943713.
Prosky, L., Asp, N., Scheweizer, T., Devries, J. y Furda, I. 1988. Determination of insoluble, soluble, and total dietary fiber in food and products. Interlaboratory study. Journal Association Official Analytical Chemist. 71: 1017-1023. PMID: 2853153

Reeves, P., Nielsen, F. y Fahey, G. 1993. AIN-93 purified diets for laboratory rodents: final report of the American Institute of Nutrition ad hoc writing committee on the reformulation of the AIN-76A rodent diet. Journal Nutrition. 123: 1939-1951. PMID: 8229312

Sánchez, I., Reynoso, C. y Salgado, L. 2015. The diet-induced metabolic syndrome is accompanied by whole-genome epigenetic changes. Genes and Nutrition. 10: 471.

SAS. Statistical Analysis System. 2004. SAS User's Guide, version 9.13, SAS Institute Inc., Cary, NC, USA.

Seema, P. 2014. Opuntia cladodes (nopal): Emerging functional food and dietary supplement. Mediterranean Journal of Nutrition and Metabolism. 7: 11-19.

Tabeshpour, J., Razavi B. y Hosseinzadeh, H. 2017. Effects of avocado (Persea americana) on metabolic syndrome: a comprehensive systematic review. Phytotherapy Research. 31: 819-837.

USDA. 2018. U.S. Department of Agriculture. Avocado. Nutrient Data Laboratory. National Nutrient Database for Standard Reference. U.S. Department of Agriculture. Washington, DC. https://ndb.nal.usda.gov/ndb/search/list.

Wang, L., Bordi, P., Fleming, J., Hill, A. y Kris, P. 2015. Effect of a moderate fat diet with and without avocados on lipoprotein particle number, size and subclasses in overweight and obese adults: a randomized controlled trial. Journal American Heart Association. 4(1). e001355. 